\title{
Research on the Narrative Form of Virtual Reality
}

\author{
Weiyin Wang \\ Communication University of China, Art Department, School of Animation and Digital Arts Animation and \\ Digital Art Institute, Beijing, China, 100024
}

Keywords: Virtual Reality, Narrative, Motion Sickness

\begin{abstract}
In recent years, virtual reality technology has been widespread concern in the community, a lot of resources have been put into the virtual reality hardware and content development and it seems that once again change the information media and human cognitive revolution is taking place. However, in recent months, the heat of the virtual reality plummeted and the reason is that there are still many problems which constraints in the current development of the virtual reality. The main research object of this paper is the narrative form of virtual reality. The article will discuss the three main problems which restricting the development of virtual reality narration, and discuss how to optimize the story experience of virtual reality world participants in the moment when hardware technology is not yet mature.
\end{abstract}

\section{Introduction}

Since ancient times, mankind has not stopped exploring the narrative. Today, the task of bearing the weight of the story seems to have fallen on the shoulders of virtual reality that people have high hopes - the aesthetic fatigue caused by print media (novels, newspapers, movies, games, etc.) perennial monopoly was replaced by the excitement of first time entering the illusionary also realistic world of the virtual reality. I believe that anyone who is shocked by the virtual reality world will be added to the fanatical attitude of this new technology greatly appreciated - after all, people want to experience their own dreams in the three-dimensional space which they created for themselves

Since 2015, the development of virtual reality industry can be described as a surge, and all this has been pushed to a climax by a large number of consumer grade headset equipment which made by big manufacturer. But after the carnival, the bottleneck of virtual reality technology gradually surfaced, forcing the market to calm down, begin to face the problems of virtual reality. How to break these constraints in the present, so that the participants can experience the most optimized story and virtual reality world will be the core issues this article try to explore and solve.

\section{The Main Bottleneck of the Current Virtual Reality Narrative}

For now, there are three main problems that restricting the development of virtual reality narrative.

Firstly, it is the dizziness problem in the virtual reality experience. It is generally believed that the sense of vertigo when using virtual reality equipment is mainly caused by motion sickness. The condition is caused by the visual information seen from the visual system doesn't match with the body movement information feel by vestibular system. To be specific, eyes caught the movement in scene but the body did not feel the movement, or eyes see a still scene but the body is feeling high speed movement, are likely to cause motion sickness. During motion sickness, the body will appear dizziness and severe cases even could have nausea or vomiting. When using virtual reality equipment, the body's range of motion constraint by the equipment and the use of space, it is difficult to match the visual information of virtual reality that motion sickness has become a high probability event. Hardware developers hope to continuously improve the display refresh rate to improve visual fluency to improve and ease the symptoms of motion sickness; and content developers can only go through with the interactive tricks to try to bypass this problem, for example, 
the way in which the participant moves is changed from running to teleport, allowing the participant to move slowly on a given track, or even not allowing the participant to move (similar to the tower defense genre), etc., to weaken the sense of speed change in a virtual reality environment, ease the contradiction between the visual system and the vestibular system, with a view to muddle through. However, even if the symptoms of motion sickness is relieved, due to the current virtual reality headset device screen cannot show a fixed depth of field effect, resulting in the eye ciliary muscle in a state of tension and cannot be relaxed. Watching the virtual reality headset screen for a long time will not only lead to eye fatigue, dizziness, and may lead to myopia.

Secondly, it is the problem that the participant's attention is distracted. Professor Jeremy Bailenson, founder and director of the Virtual Human Interaction Lab of Stanford University, said in an interview: "So the challenge of storytelling is, there is this thing called director, she is a brilliant person that tells you where to look, when to look by moving the camera. In VR, there's this very cool lighting here, in general it makes you special that you get to do whatever you want. "As Professor Bailenson puts it, virtual reality is not a medium suitable for a linear narrative structure In virtual reality, the participant can arbitrarily look at any direction of a 720 degrees space, so it may miss an important story because of an insignificant detail, which in turn destroys the complete experience of the whole linear narrative. In addition, because the participants viewed the virtual world from a free perspective, make editing a virtual reality film almost impossible. Imagine that after the end of a scenario, the participants suddenly found a piece of interest in the virtual world. When he watched the item with great interest, the whole world suddenly turned black and then switched to other scenes, and the items in front of them disappeared ... How big devastating blow could cause to the immersive experience of the participants?

Finally, the Swayze effect is subtler than the previous two restrictions. This is the virtual reality experience that Oculus's Story Studio team has named after Patrick Wayne Swayze, hero from the 1990 film $<$ Ghost $>$. In the play, Patrick was killed and returned as a wandering soul and he tried to interact with the physical world to attract people's attention. But because the law of the two worlds is different, no matter how hard he tried he always failed to succeed. This effect will also appear in the virtual reality story experience (especially only the virtual reality video which only allow participant's perspective to turn) - the participants feel themselves exist in the virtual world, but the characters of the virtual world cannot recognize the existence of the participants. In this case, the more closed of a film's story line, the more "exclusion" participants will feel. Participants will feel the role of the film in their own words and their own existence was ignored, and then have a sense of exclusion inexplicable sense of loss. Most of the participants have a deep understanding of this feeling when experiencing Story Studio's two virtual reality cartoons <Lost $>$ (2015) and $<$ Henry $>$ (2016), especially in <Henry $>$. The hero in $<$ Henry $>$ is a hedgehog called Henry, he particularly likes to embrace others, so stabbed a lot of animals, thus all the other animals keeps a distance from him, makes he have no friend at all. At his birthday party, he made a wish to have a good friend. Throughout the watching experience, Henry will have eye contact with the participant several times, the participants will feel a strong emotional connection is established with the virtual character at this time; However, when Henry have the expression he still feels lonely, this emotional connection was immediately torn off. Participants began to question: "If you can perceive my presence, why do you still feel lonely?" In this way, the early immersion experience was instantly destroyed. While the virtual reality attempt to break down the "fourth wall", it still declared a failure at this moment. Thus, even Story Studio comes up with the question "perhaps presence and narrative cancel each other out”.

Three problems above are just huge "iceberg" which emerged on the way of narrative in virtual reality I believe there still have countless obstacles ahead waiting for developers. Facing so many unknown matters, how can we optimize the participants' virtual reality story experience? 


\section{Build a World}

Possibility is perhaps the best weapon against unknowns.

I believe that if the use of fragmental narrative structure should be able to greatly optimize the narrative experience of virtual reality at this stage. Different from the linear and most of the tree-like non-linear narrative structure, fragmental narrative structure breaks the concept of time flow, information of the plot are broken into pieces of debris, hidden in the virtual space, waiting for the participants to explore and excavation. These fragments can be in any form: a mark on the banner, a hidden inscription, an incomplete letter, a whispering word ... ... the amount of information revealed by one or two fragments is limited and hazy, and even let the participants feel puzzled; but as the participants find more and more fragments in the process of exploration of the virtual world, the debris between the relevance will gradually clear up. When participants put the fragments together, a "map" will emerge, the "truth" of virtual world and the legendary stories hidden in the world will gradually surfaced.

The structure of the fragmental narrative is quite different from the common narrative structures of movies, novels, plays and even games. As shown in Figure 1, it is different from the art form that is mainly narrated by action and dialogue. The fragmental narrative structure is centered on the whole virtual world. The creator conceals a piece of fragmented clue information in the virtual world, waiting for the participants to find them. When a certain number of clues are found, these clues will act on the minds of the participants, so that they will break the broken clues in the brain into a complete "puzzle" to understand the world's "real" form and the story in which plot. "In general, the user may have to put the pieces together to understand the whole story." However, this is only a narrative aspect of the fragmental narrative structure - the inner narrative, which is only used in the individual narrative of the participants. The narrative did not end at this level, but with the participants as the center, radiating out another narrative - outer narrative (Figure 2). Participants are often dissatisfied with their own interpretation of the fragmented clue information, so they will seek help or identity - discuss with the other participants on the social network and work together to improve the information of the virtual world. At this time, the identity of the participant has been changed from an observer and a listener to a narrator who participates in narrative and constructs a virtual world. If we see each participant as a piece of debris in a whole piece of narrative puzzles, then all the "participants narrative fragments" can form a more grand large narrative system. Through the fragmental narrative structure, not only the participants enjoy the fun to bring their' wisdom into play, the virtual world's influence and its vitality have also been greatly extended. At this point, we "no longer need a story, or no longer need a pre-designed story, need to design that is the story of the world, is the interaction with the rules of the world itself --has an interactive world, Interactive rules and user interaction, naturally there will be many stories from which to be created."

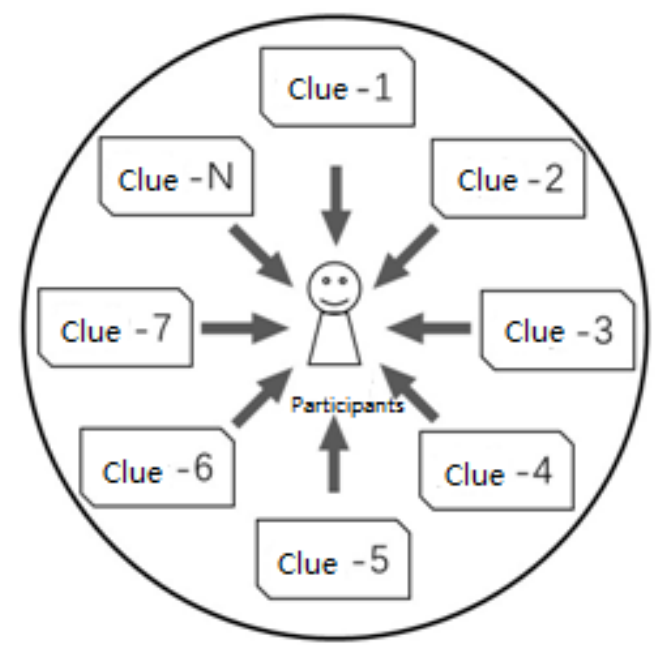

Figure 1 The inner narrative of the fragmental narrative structure 


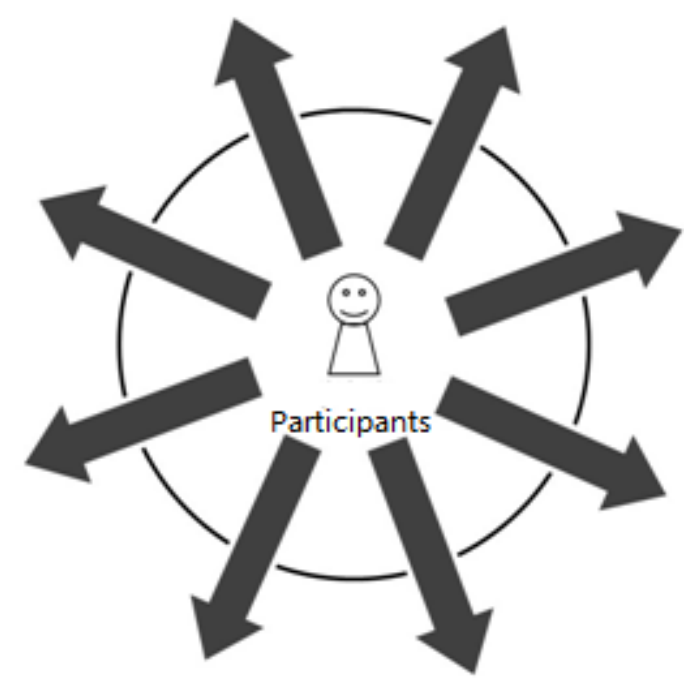

Figure 2 The outer narrative of the fragmental narrative structure

If the linear narrative structure is using time stream to unfold the plot, then the fragmental narrative structure is using space to tell the story. The former is in the pursuit of the certainty and subjectivity of the narrative, while the later emphasizes the possibility and objectivity of narrative. Under the fragmental narrative structure, the virtual world is the story itself, and the participant not just experience the story, he also participated in the construction process of the world and became a narrator of the same status as the original author of the virtual world. The importance and initiative of the participants have been improved, and the identity and the power of the original author are limited or even completely removed. At this point, the participants can fully control their own rhythm and speed of exploration, can effectively alleviate the discomfort caused by motion sickness; fragmented information is also fully using the participants' scattered attention, encourage them to distract attention everywhere to explore as many clues as possible. Fragmental information also means that the participants can stop at any time according to their own wishes to rest, not because it is in a large plot and not willing to stop. Since then the narrative works successfully broke through the restrictions of "fourth wall", and truly become works without center to achieve the long-cherished wish of literary and film industry - digestive implied author.

As an art form of time and space at the same time, the narrative experience of drama may be more fit virtual reality compared to the film and the novel. The drama <Sleep no more> is a typical fragmental narrative structure. The stage is a building with five floors. The whole play time is three hours, during which there will be several series of plot lines in the whole building in different spaces at the same time. In addition, the building also placed a large number of documents, and the information carried by documents help to fill, improve the role of the story background. During the performance, the audience can walk around in the building, out of the room, read the information. Because the use of a special structure, so that the audience cannot glimpse the story of the whole picture all at once, to gradually understand the story requires audiences repeatedly watching it for many times or exchange watching experience with others. This unique experience, which distinguishes it from traditional drama, is well received.

Not come singly but in pairs, the video game <Bloodborne $>$ (2015) has also received a wide acclaim from the market because of the use of fragmental narrative structures. There is no linear story in the game (in this case it does more thoroughly than the <Sleep no more>), players can only guess what happened in this world through the sporadic clue - The items obtained in the game props have a short and seem to elaborate text introduction, and some may be the introduction of the function of the items, and some are the introduction of the origin of the items; game NPC (non-player character) dialogue is also very simple and vague. This form of narrative structure does not lead to the player's resentment because of lack of certainty, but it given the player with enough imagination because of its large area of "blank", so that they can use imagination to complement the story and thus get widely praised by the players. Almost every post on social network about 
$<$ Bloodborne $>$ story can lead to intense discussion and debate, making the game's influence extended to a broader field invisibly.

Japanese company Capcom released <Biohazard 7> on January 24, 2017. The Playstation4 version game added Playstation VR mode, so that players can play with it by wearing virtual reality headset equipment. Interestingly, as a well-known manufacturer produced by the highly anticipated works, the game is also taking the fragmental narrative structure: at first the game uses a large number of fragmental information to create a virtual space which full of strange atmosphere and suspense for the players. Next, the players need to continue fight and solve puzzles in the game, and through the gradual acquisition of clues to guess the truth behind the game. In addition to the fragmental narrative structure, the most commendable is the exploration of editing method for the virtual reality in the game: players can find a few videotapes in the game, after playing the video, the player's identity will be switched from the actor to the role in the video, then make interaction and exploration in the perspective of the character in the video. This kind of editing method perfectly conceals the identity of the producer, gives full control to the player's subjective initiative, allowing the player to switch the image space autonomously, thus solving the conflict between player's attention and the editing. $<$ Biohazard $7>$ is undoubtedly setting a tonic into the virtual reality industry, I believe the future will have more virtual reality narrative works to explore and innovation based on it.

\section{Conclusion}

Whether it is a linear or non-linear narrative structure, whether it is movies, novels, plays or games, no matter how magnificent narrative, how beautiful rhetoric, once the plot is exhausted, after all, it is like a burning candle, and it is difficult to ignite the user's passion. But the virtual reality world which organically fuses the fragmental narrative structure is a completely different concept because it reduces the human exploration and understanding of the world by creating a "wandering world". Based on such a virtual world, with the fermentation of social network, it is more likely to produce grand narrative system beyond our imagination.

Although it is wearing a lot of shackles at birth, the future of virtual reality is still worthy of our expectations. Through the virtual reality, we will be able to push the image narrative to the highest level " There's an end to the words, but not to their message ".

\section{References}

[1] Qin Lanjun: "Interaction and Story: VR's Narrative Ecology" [J], Literature and Art Studies, 2016, No. 12.

[2] Jiang Zhulang: "Explore the VR works on the implied author of the impact" [J], contemporary film, 2016, No. 12. 